\title{
Preoperative high dose rate brachytherapy for clinical stage II endometrial carcinoma
}

\author{
Gaurav Shukla, PhD', Sushil Beriwal, MD², Thomas C. Krivak, MD², Joseph L. Kelley, MD³, Paniti Sukumvanich, MD³, \\ Robert P. Edwards, MD³, Scott Richard, MD³, Alexander Olawaiye, MD³ , Kristin K. Zorn, MD³ \\ University of Pittsburgh School of Medicine, 2Department of Radiation Oncology, University of Pittsburgh Cancer Institute. \\ 3Department of Gynecologic Oncology, University of Pittsburgh Medical Center, USA
}

\begin{abstract}
Purpose: We sought to evaluate pathological response, tolerance, and outcome after preoperative (neoadjuvant) high dose rate brachytherapy in a small series of patients with clinical stage II endometrial carcinoma, and to evaluate a dose and fractionation protocol for this treatment.

Material and methods: Twelve women diagnosed with clinical stage II endometrial carcinoma from 1999-2010 were treated with preoperative radiation therapy. Their medical charts were retrospectively analyzed for HDR treatment regimen, pathological response, and longitudinal outcomes. Radiation doses were normalized to a biologically equivalent dose of 2 Gy per fraction (EQD2).

Results: Two patients had complete pathological response to neoadjuvant therapy; five more had only microscopic residual disease at the time of surgery. At a median follow up of 37 months (1-91 months), one patient has developed recurrence at the vaginal apex six months after completing initial therapy, while another developed a lung recurrence at 28 months. Two-year disease-free and cause-specific survivals were $88 \%$ and $100 \%$, respectively.

Conclusions: Our small study shows that the HDR fractionation schedule, as done in our series for preoperative radiation therapy for clinical stage II endometrial cancer, is well tolerated and would be an option for patients treated with neoadjuvant radiation therapy.

Key words: endometrial carcinoma, neoadjuvant, radiation, HDR brachytherapy.

\section{Purpose}

Endometrial carcinoma is the most common gynecologic malignancy, with an estimated 40100 incident cases and 7470 deaths in 2009 [1]. The current standard of care for patients with endometrial cancer consists of surgical staging, including total hysterectomy, bilateral salpingo-oophorectomy, and pelvic and para-aortic lymph node dissection. Depending on the surgical stage and histological grade, the adjuvant treatment may include radiation therapy and/or chemotherapy [2].

In approximately $10 \%$ of patients, endometrial carcinoma involves the cervix [3-6]. However, there is a relative lack of consensus regarding the most appropriate management strategy for these patients. Patients with clinically occult stage II disease usually have undergone simple hysterectomy rather than radical hysterectomy, which is then followed by adjuvant radiation therapy. Patients with clinical stage II disease may be treated with preoperative (neoadjuvant) radiation therapy followed by simple hysterectomy, or alternatively, primary radical hysterectomy and possible adjuvant tumor-directed radiation therapy.
Neoadjuvant treatment involves a combination of external beam radiation and intracavitary brachytherapy. It is especially advocated for patients having co-morbidities that limit their candidacy for radical surgery, including obesity. In all patients with clinical stage II disease, neoadjuvant radiotherapy can decrease the size and extent of bulky cervical disease, making it easier to obtain clear surgical margins. Further, it avoids the increased morbidity associated with lymphadenectomy followed by radiation therapy, as is seen in cervical cancer [7]. Conversely, advocates of primary treatment by radical hysterectomy cite the ability to debulk large tumor prior to attempting radiation therapy, to clarify the origin of the primary tumor as cervical or endometrial, and to clarify the stage and grade of disease prior to initiating adjuvant therapy [8].

Most of the published literature on preoperative treatment is with low dose rate (LDR) brachytherapy [9-12]. Due to the relative rarity of this clinical scenario, there is no published data on dose, fractionation or pathological response to high dose rate (HDR) intracavitary brachytherapy in the neoadjuvant setting. The goal of this study was to evaluate pathological response, tolerance, and outcome with this latter approach. 


\section{Material and methods}

Twelve women clinically diagnosed with clinical stage II endometrial carcinoma from 1999-2010 were treated with preoperative radiation therapy. Their treatments and outcomes were analyzed and presented in Table 1 . All patients had biopsy-confirmed endometrial cancer and had pretreatment CT scan and/ or MRI for staging. The dose of external beam (EBRT) was 45-50.4 Gy in 25 to 28 fractions followed by HDR brachytherapy. The HDR brachytherapy was done with placement of a Smit sleeve and tandem and ring applicator. Prior to 2004, three patients received a dose of $4 \mathrm{~Gy}$ in four fractions, but since that time all patients received doses between 5-5.5 Gy in three fractions. Initially, for the first six patients, the dose was prescribed to point A with orthogonal film based dosimetry. The last six patients were treated with MRI or CT based planning with the clinical target volume being the entire uterus and cervix (Fig. 1).

Simple hysterectomy with lymph node sampling was performed four to eight weeks after the last brachytherapy treatment. The pathological response and outcome data were extracted and analyzed. The total doses from EBRT and brachytherapy were summated and normalized to a biologically equivalent dose of two Gy per fraction (EQD2) using the linear quadratic model and $\frac{\alpha}{\beta} 10$ Gy [13]. All patients were followed every three months for the first two years, and every six months thereafter, with vaginal cytology at each surveillance visit. Follow-up data were analyzed using PASW version 18.

\section{Results}

The demographics, histology, and response data are as described in Table 1 . The median age was 57 (range 41-76) with median body mass index (BMI) of 35 (range 29-45). All patients completed the planned course of preoperative radiation therapy with a median EQD2 dose of 65.0 Gy (range 56.3-75.4 Gy). No patient developed acute grade three or higher toxicity.

All patients had simple hysterectomy with lymph node sampling with uneventful recovery from the operation. Complete pathological response ( $\mathrm{pCR}$ ) was seen in two patients $(17 \%)$ while five patients $(42 \%)$ had only microscopic residual disease confined to either the endometrium or cervical glands. Two patients were found to have para-aortic disease and received adjuvant para-aortic radiation. Two patients with high grade pathology (one each with serous and clear cell histology) also received adjuvant chemotherapy after surgery. At a median follow up of 37 months (1-91 months), one patient has developed recurrence at the vaginal apex six months after completing initial therapy and is alive with the disease at 91 months, while another developed a lung recurrence at 28 months. One patient developed a second primary lung carcinoma that was treated surgically. One patient has died of comorbidities at 86 months with no evidence of the disease at last follow up. No patient developed late grade three or higher radiation treatment-related morbidity. Two-year actuarial disease-free and cause-specific survivals were $88 \%$ and $100 \%$, respectively; these were calculated for only those patients with at least two years of follow-up.

\section{Discussion}

Preoperative radiation therapy is one of the options for clinical stage II endometrial cancer. The goals of this neoadjuvant therapy are to shrink the primary disease, to treat pelvic nodes and parametria, and to make the patient more

Table 1. Demographics, tumor histology, and pathological response to neoadjuvant HDR brachytherapy prior to simple hysterectomy in twelve patients with clinical stage II endometrial carcinoma

\begin{tabular}{|c|c|c|c|c|c|c|c|c|}
\hline Pt & Age & Histology & EQD2 (Gy) & $\begin{array}{l}\text { Pathological response } \\
\text { (partial or complete) }\end{array}$ & FIGO grade & $\begin{array}{c}\text { FIGO stage } \\
\text { (pathological) }\end{array}$ & $\begin{array}{c}\text { Follow up } \\
\text { (month) }\end{array}$ & $\begin{array}{l}\text { Outcome at last } \\
\text { follow up }\end{array}$ \\
\hline 1 & 64 & Endometrioid & 63.75 & $P R^{*}$ & 2 & ॥ & 55 & NED \\
\hline 2 & 50 & Endometrioid & 63.75 & PR & 3 & ॥ & 50 & NED \\
\hline 3 & 64 & Endometrioid & 59 & $P R$ & 1 & II & 86 & Deceased \\
\hline 4 & 60 & $\begin{array}{l}\text { Mixed endometrioid } \\
\text { and clear cell }\end{array}$ & 66.31 & $P R^{*}$ & 3 & IA & 22 & NED \\
\hline $5^{\dagger}$ & 49 & Endometrioid & 63.75 & $C R$ & 3 & 0 & 23 & NED \\
\hline $6^{+}$ & 53 & $\begin{array}{l}\text { Mixed endometrioid } \\
\text { and serous }\end{array}$ & 66.31 & $C R$ & 3 & 0 & 3 & NED \\
\hline 7 & 76 & Endometrioid & 75.4 & $P R$ & 3 & ॥ & 73 & $\begin{array}{l}\text { NED, } 2^{\text {nd }} \\
\text { primary ca }\end{array}$ \\
\hline 8 & 72 & Endometrioid & 70 & $P R^{*}$ & 1 & IIIC & 51 & NED \\
\hline 9 & 66 & Endometrioid & 70 & $\mathrm{PR}$ & 2 & IIIC & 91 & AWD \\
\hline 10 & 41 & Endometrioid & 67.2 & $P R$ & 1 & II & 1 & NED \\
\hline 11 & 50 & Endometrioid & 63.63 & $P R^{*}$ & 1 & ॥ & 9 & NED \\
\hline 12 & 50 & Endometrioid & 56.29 & $P R^{*}$ & 2 & II & 1 & NED \\
\hline
\end{tabular}

†Histological and FIGO grading from endometrial biopsy, not surgical specimen, EQD2 - Equivalent Dose in 2-Gray increments, PR - partial response, $C R$ - complete response, $P R^{*}$ - microscopic residual disease, NED - no evidence of disease, AWD - alive with disease 

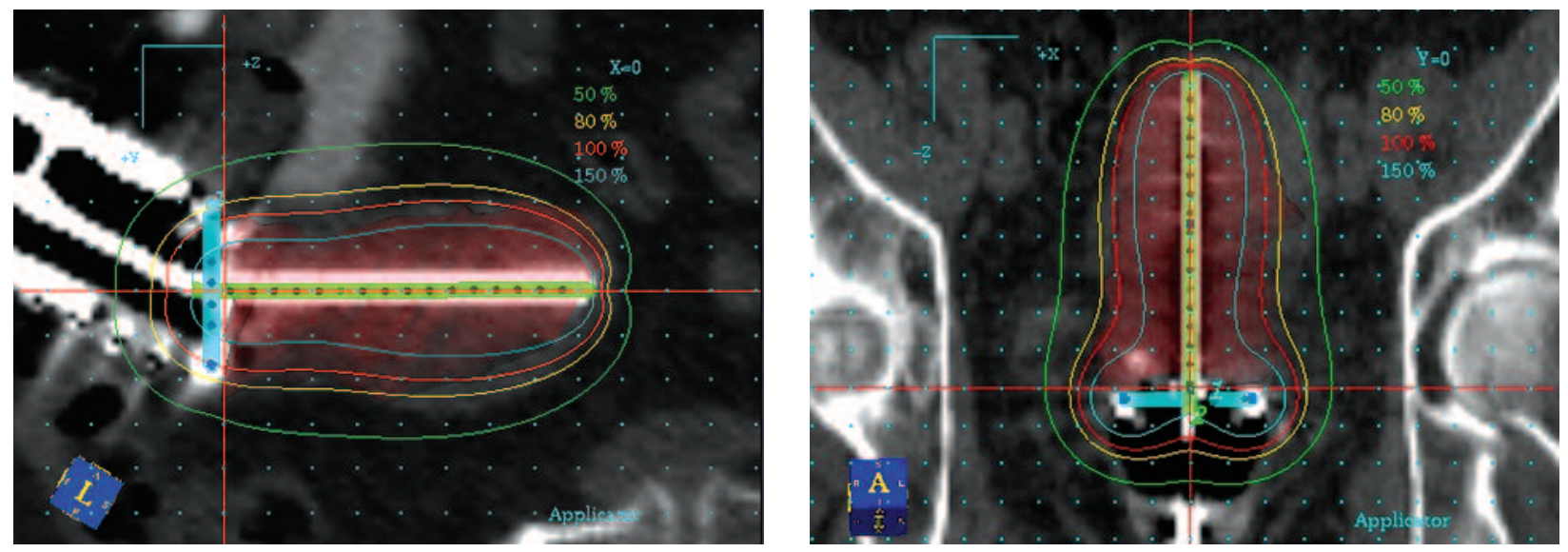

Fig. 1. Sagittal (left) and coronal (right) view showing clinical target volume (red) covered by prescription isodose line of $100 \%$ (red)

suitable for simple hysterectomy. Historically, brachytherapy has been performed using an LDR technique [9-12]. There is no published experience with HDR intracavitary brachytherapy as preoperative treatment for endometrial cancer. The American Brachytherapy Society in its published guidelines for HDR brachytherapy for endometrial cancer have not recommended a dose or fractionation schedule for preoperative HDR brachytherapy for stage II endometrial cancer, likely due to the lack of published data [2].

Multiple studies have shown equivalency of HDR and LDR brachytherapy for cervical cancer [14, 15]. The use of HDR brachytherapy has increased significantly over the last decade [16]. The advantages of HDR include short treatment time, ability for optimization, and a lack of radiation exposure to healthcare personnel. The disadvantages include multiple insertions and the deficiency of a standardized fractionation schedule. Since 2004, our institution has followed a fractionation schedule of three fractions of 5-5.5 Gy to deliver a dose equivalent to 65-70 Gy. The schedule has been well tolerated, has shown a good response rate, and has not revealed significant morbidity. More recently, we have integrated three-dimensional planning for HDR brachytherapy, which helps to conform better the dose to the tumor while reducing the dose to critical organs (Fig. 1).

The results of this small series are comparable to published studies using LDR brachytherapy. In a series of 74 patients with stage II endometrial cancer treated at the University of Kentucky (UK) with a combined dose of $65 \mathrm{~Gy}$, the complete pathological response rate was seen in $31 \%$ and the residual disease confined to endometrium in $29 \%[10,11]$. In our study, which had a median EQD2 dose of $66.31 \mathrm{~Gy}, \mathrm{pCR}$ was seen in $22 \%$, with superficial residual disease confined to the endometrium in $33 \%$. In the UK series, para-aortic nodal disease was noted at the time of surgery in five patients, compared to two patients in our series. Patients with cervical involvement are at increased risk of both pelvic and para-aortic nodal involvement; since only the pelvic nodes are treated during the whole pelvic radiation therapy given preoperatively, para-aortic nodal sampling at the time of surgery should be considered [17].

The optimal management for clinical stage II disease that provides a better outcome, in terms of disease control and morbidity, is not known. It is difficult to study prospectively because of the comparative rarity of this clinical scenario. In a retrospective analysis of 184 consecutive patients with clinical or pathologic stage II endometrial cancer treated with definitive intent with either preoperative or postoperative radiation therapy, only the grade and histology were found to be independent predictors of disease free survival [18]. The timing of radiotherapy was not an independent predictor of the outcome.

\section{Conclusions}

Since a subset of these patients are not ideal candidates for radical surgery because of medical co-morbidities, advanced age, and obesity, the preoperative radiation therapy followed by simple hysterectomy may be a better option [6]. Ours is the first published series using HDR and shows the HDR fractionation schedule, as done in our series, is well tolerated and would be an option for patients treated with neoadjuvant radiation therapy.

\section{References}

1. Jemal A, Siegel R, Ward E et al. Cancer Statistics, 2009. CA Cancer J Clin 2009; 59: 225-249.

2. Nag S, Erickson B, Parikh S et al. The American Brachytherapy Society recommendations for high-dose-rate brachytherapy for carcinoma of the endometrium. Int J Radiat Oncol Biol Phys 2000; 48: 779-790.

3. Fayed A, Mutch DG, Rader JS et al. Comparison of high-doserate and low-dose-rate brachytherapy in the treatment of endometrial carcinoma. Int J Radiat Oncol Biol Phys 2007; 67: 480-484.

4. Keys HM, Roberts JA, Brunetto VL et al. A phase III trial of surgery with or without adjunctive external pelvic radiation therapy in intermediate risk endometrial adenocarcinoma: a Gynecologic Oncology group study. Gynecol Oncol 2004; 92: 744-751.

5. Small W Jr, Erickson B, Kwakwa F. American Brachytherapy Society survey regarding practice patterns of postoperative irradiation for endometrial cancer: current status of vaginal brachytherapy. Int J Radiat Oncol Biol Phys 2005; 63: 1502-1507.

6. Wright JD, Fiorelli J, Kansler AL et al. Optimizing the management of stage II endometrial cancer: the role of radical hysterectomy and radiation. Am J Obstet Gynecol 2009; 200: 419. e1-7. 
7. Manchana T, Sirisabya N, Lertkhachonsuk R et al. Long term complications after radical hysterectomy with pelvic lymphadenectomy. J Med Assoc Thai 2009; 92: 451-456.

8. Sartori E, Gadducci A, Landoni F et al. Clinical behavior of 203 stage II endometrial cancer cases: the impact of primary surgical approach and of adjuvant radiation therapy. Int J Gynecol Cancer 2001; 11: 430-437.

9. Reisinger SA, Staros EB, Feld R et al. Preoperative radiation therapy in clinical stage II endometrial carcinoma. Gynecol Oncol 1992; 45: 174-178.

10. Maruyama Y, Yoneda J, Coffey C et al. Tandem-vaginal cylinder applicator for radiation therapy of uterine adenocarcinoma. Radiother Oncol 1992; 25: 140-141.

11. Higgins RV, van Nagell JR Jr, Horn EJ et al. Preoperative radiation therapy followed by extrafascial hysterectomy in patients with stage II endometrial carcinoma. Cancer 1991; 68: 1261 1264.

12. Komaki R, Cox JD, Hartz A et al. Influence of preoperative irradiation on failures of endometrial carcinoma with high risk of lymph node metastasis. Am J Clin Oncol 1984; 7: 661-668.

13. Joiner MC, van der Kogel AJ. The linear-quadratic approach to fractionation and calculation of isoeffect relationships. In: Steel GG (ed.). Basic clinical radiobiology. 2nd ed. A Hodder Arnold Publication, London 1997; pp. 106-122.

14. Viani GA, Manta GB, Stefano EJ et al. Brachytherapy for cervix cancer: low-dose rate or high-dose rate brachytherapy a meta-analysis of clinical trials. J Exp Clin Cancer Res 2009; 28: 47.

15. Nag S, Orton C, Young D et al. The American brachytherapy society survey of brachytherapy practice for carcinoma of the cervix in the United States. Gynecol Oncol 1999; 73: 111-118.

16. Pearce A, Craighead P, Kay I et al. Brachytherapy for carcinoma of the cervix: a Canadian survey of practice patterns in a changing era. Radiother Oncol 2009; 91: 194-196.

17. Creasman WT, Morrow CP, Bundy BN et al. Surgical pathologic spread patterns of endometrial cancer. A Gynecologic Oncology Group Study. Cancer 1987; 60: 2035-2041.

18. Lanciano RM, Curran WJ Jr, Greven KM et al. Influence of grade, histologic subtype, and timing of radiotherapy on outcome among patients with stage II carcinoma of the endometrium. Gynecol Oncol 1990; 39: 368-373. 